\title{
Effective field theories for muonic hydrogen
}

\author{
Clara Peset ${ }^{1, a}$ \\ ${ }^{1}$ Grup de Física Teòrica, Dept. Física and IFAE-BIST, Universitat Autònoma de Barcelona, E-08193 Bel- \\ laterra (Barcelona), Spain
}

\begin{abstract}
Experimental measurements of muonic hydrogen bound states have recently started to take place and provide a powerful setting in which to study the properties of QCD. We profit from the power of effective field theories (EFTs) to provide a theoretical framework in which to study muonic hydrogen in a model independent fashion. In particular, we compute expressions for the Lamb shift and the hyperfine splitting. These expressions include the leading logarithmic $O\left(m_{\mu} \alpha^{6}\right)$ terms, as well as the leading $O\left(m_{\mu} \alpha^{5} \frac{m_{\mu}^{2}}{\Lambda_{\mathrm{OCD}}^{2}}\right)$ hadronic effects. Most remarkably, our analyses include the determination of the spin-dependent and spin-independent structure functions of the forward virtualphoton Compton tensor of the proton to $O\left(p^{3}\right)$ in HBET and including the Delta particle. Using these results we obtain the leading hadronic contributions to the Wilson coefficients of the lepton-proton four fermion operators in NRQED. The spin-independent coefficient yields a pure prediction for the two-photon exchange contribution to the muonic hydrogen Lamb shift, which is the main source of uncertainty in our computation. The spindependent coefficient yields the prediction of the hyperfine splitting. The use of EFTs crucially helps us organizing the computation, in such a way that we can clearly address the parametric accuracy of our result. Furthermore, we review in the context of NRQED all the contributions to the energy shift of $O\left(m_{\mu} \alpha^{5}\right)$, as well as those that scale like $m_{r} \alpha^{6} \times$ logarithms.
\end{abstract}

\section{Introduction}

In these proceedings we study those systems composed by a lepton and a proton which are weakly bounded by the electromagnetic force, and in particular muonic hydrogen. Hadronic information is encoded in the interaction of the baryon and the lepton it is bounded to, when one takes into account the effects of the hadron's finite size. Therefore, they are a good place to learn about the physics inside hadrons upon comparison with experimental measurements. To do so, we develop the appropriate effective field theory (EFT), specifically dedicated to describing the interval of the energy range in which the relevant dynamics of the strong sector dominates.

The development of quantum field theories for baryons at low energies is closely related to the approximate chiral symmetry of the QCD Lagrangian. The EFT description of baryons in this picture is problematic, since their mass is comparable to the hadronization scale $\Lambda_{\mathrm{QCD}}$, thus reaching the limit of validity of the chiral expansion [1]. The inclusion of the heavy mass methods overcomes this issue

ae-mail: peset@ifae.es 
by going to the nonrelativistic (NR) limit and provides well defined power counting rules [2, 3]. this is known as heavy baryon chiral perturbation theory (HBChPT). In this setting, we include the Delta particle $\Delta(1232)$, which is only $\sim 300 \mathrm{MeV}$ away from the nucleon mass and couples strongly to the pion-nucleon sector. On top of this, an outcome of applying large- $N_{c}$ rationale to baryons is that the proton and the Delta particle become degenerate in mass.

In fact, bound states are naturally nonrelativistic systems, since the relative velocity between their components $v$ is small and their typical scales relate as $m_{r} \gg|\mathbf{p}| \gg E$. The EFT approach to describe the dynamics of NR systems allows us to obtain model independent predictions which can be organized in an efficient and systematic way (due to the well defined power counting rules), thus yielding a unified framework to determine the nonperturbative effects. When the system is bounded by QED, the only expansion parameter of the theory is the electromagnetic coupling constant, which is of the order of the velocity $\alpha \sim v$. Therefore the typical energy scales in the system scale as follows:

\section{Scales in bound state Coulomb interaction \\ Hard scale: $m_{r} \quad \longrightarrow \quad m_{r}$ \\ Soft scale: $|\mathbf{p}| \quad \longrightarrow \quad m_{r} \alpha$ \\ Ultrasoft scale: $E \quad \longrightarrow \quad m_{r} \alpha^{2}$}

It is then clear that the scales are well separated, and thus, since we are interested in the low energy regime, we can first integrate out the hard scale to obtain NRQED [4]. Furthermore, for the physics of our interest we can also integrate out the soft scales thus obtaining a theory for ultrasoft photons, namely pNRQED [5]. This theory has the proper degrees of freedom to describe systems such as positronium, muonium, hydrogen, muonic hydrogen, etc.

\section{Muonic Hydrogen}

Given the recent and the foreseen experimental measurements of muonic hydrogen states [6-8], a thorough theoretical study of this system is needed. We perform it in an EFT framework, and the results can be found in full detail in [9-12].

When dealing with muonic hydrogen, the EFT organization is specially useful, as its dynamics is characterized by several scales:

$$
m_{\rho} \sim m_{p}, \quad m_{\mu} \sim m_{\pi} \sim m_{r}, \quad m_{r} \alpha \sim m_{e}
$$

From the ratios of these scales, we can obtain small expansion parameters:

$$
\frac{m_{\pi}}{m_{p}} \sim \frac{m_{\mu}}{m_{p}} \approx \frac{1}{9}, \frac{m_{e}}{m_{r}} \sim \frac{m_{r} \alpha}{m_{r}} \sim \alpha \approx \frac{1}{137}
$$

Thus, we can organize the energy levels of muonic hydrogen as an expansion in the coupling $\alpha$, with coefficients that contain at the same time an expansion in the aforementioned parameters. Schematically this is:

$$
E^{\mu p}=\frac{-m_{r} \alpha^{2}}{2 n^{2}}\left(1+c_{1} \alpha+c_{2} \alpha^{2}+\ldots\right)
$$


where $c_{1} \sim c_{1}\left[\frac{\mu}{m_{e}}\right]$ is a pure QED-like coefficient, and for $n>1$,

$$
c_{n} \sim \sum_{j=0}^{\infty} c_{n}^{(j)}\left(\frac{m_{\pi}}{m_{p}}\right)^{j}, \quad \text { with } \quad c_{n}^{(j)} \sim c_{n}^{(j)}\left[\frac{m_{r}}{m_{\mu}}, \frac{m_{\mu}}{m_{\pi}}, \ldots\right]
$$

already comprise information of the hadronic scales.

In order to obtain the theory where $E \sim m_{r} \alpha^{2}$ (pNRQED), every degree of freedom with larger energy is integrated out. This implies treating the proton and muon in a NR fashion and integrating out pions and Delta particles. This is the step of going from HBChPT to NRQED. By integrating out the scale $m_{r} \alpha$, pNRQED is obtained and the potentials appear. Schematically the path followed is $\left(\Delta \equiv m_{\Delta}-m_{p}\right)$ :

$$
\text { HBChPT } \stackrel{\left(m_{\pi / \mu}, \Delta\right)}{\Longrightarrow} \text { NRQED } \stackrel{\left(m_{\mu} \alpha\right)}{\Longrightarrow} \text { pNRQED . }
$$

Through this procedure the specific pNRQED Lagrangian up to $O\left(m_{r} \alpha^{5}\right)$ is obtained [13]:

$$
\begin{aligned}
& L_{\mathrm{PNRQED}}=\int d^{3} \mathbf{x} d^{3} \mathbf{X} S^{\dagger}(\mathbf{x}, \mathbf{X}, t)\left\{i \partial_{0}-\frac{\mathbf{p}^{2}}{2 m_{r}}+\frac{\mathbf{p}^{4}}{8 m_{\mu}^{3}}+\frac{\mathbf{p}^{4}}{8 m_{p}^{3}}-\frac{\mathbf{P}^{2}}{2 M}\right. \\
& \left.-V\left(\mathbf{x}, \mathbf{p}, \sigma_{1}, \sigma_{2}\right)+e\left(\frac{Z_{\mu} m_{p}+Z_{p} m_{\mu}}{m_{p}+m_{\mu}}\right) \mathbf{x} \cdot \mathbf{E}(\mathbf{X}, t)\right\} S(\mathbf{x}, \mathbf{X}, t)-\int d^{3} \mathbf{X}_{4}^{1} F_{\mu \nu} F^{\mu \nu}
\end{aligned}
$$

Of special relevance for our computations is the potential term of the Lagrangian. In order to properly organize our computation, we split it in terms of its main powers of $\alpha$ and the constituent masses:

$$
V\left(\mathbf{x}, \mathbf{p}, \sigma_{1}, \sigma_{2}\right)=V^{(0)}(r)+V^{(1)}(r)+V^{(2)}(r)+\cdots,
$$

where we define

$$
V^{(n)} \propto \frac{1}{m_{\mu}^{n}} \quad \text { and } \quad V^{(n, r)} \propto \frac{1}{m_{\mu}^{n}} \alpha^{r}+\text { expansions in small parameters. }
$$

We will obtain the relevant contributions to the energy levels from these potentials in order to compute the Lamb shift $(2 \mathrm{P} \longrightarrow 2 \mathrm{~S}$ transition), and the $2 \mathrm{~S}$ hyperfine splitting in muonic hydrogen. Both of these energy shifts have been measured experimentally $[6,14]$ allowing us to test the predictive strength of our theory.

\subsection{The Lamb shift and the proton radius}

In order to be competitive with the experimental errors we obtain a theoretical prediction for the Lamb shift with precision of $O\left(m_{\mu} \alpha^{5}\right)$ in the counting of the pure QED contributions and $O\left(m_{\mu} \alpha^{5} \frac{m_{\mu}^{2}}{m_{\rho}^{2}}\right)$ in the hadronic ones. The final result of our analysis yields

$$
\Delta E_{L S}=206.0243(30)-5.2270(7) \frac{r_{p}^{2}}{\mathrm{fm}^{2}}+0.0455(125)+O\left(m_{\mu} \alpha^{5} \frac{m_{\mu}^{3}}{m_{\rho}^{3}}, m_{\mu} \alpha^{6}\right) \mathrm{meV},
$$

where the first number is the prediction arising from pure QED considerations and the last two encode the hadronic effects. Note that Eq. (8) allows us to obtain a value for the proton charge radius 
upon comparison with experiment. This is of special relevance as to this today there exists a $\sim 7 \sigma$ discrepancy between the measurement of this quantity in muonic hydrogen and its previous CODATA determination coming from hydrogen spectroscopy and $e p$-scattering [15].

In our EFT setting, the hadronic effects are encoded in the $\delta^{(3)}(r)$-potential of pNRQED. Moreover, the proton radius is encoded in the Wilson coefficient

$$
c_{D, \overline{\mathrm{MS}}}^{(p)}(v) \equiv Z_{p}+\frac{4}{3} \frac{Z_{p}^{3} \alpha}{\pi} \ln \left(\frac{m_{p}^{2}}{v^{2}}\right)+\frac{4}{3} r_{p}^{2} m_{p}^{2}+O\left(\alpha^{2}\right)
$$

and therefore it is a well-defined quantity.

We devote most of our effort to the hadronic effects that arise from the two photon exchange (TPE) in the last term in Eq. (8). They start at $O\left(m_{\mu} \alpha^{4}\right)$ and they are the main source of the theoretical uncertainty. This is different from the analysis in electronic hydrogen, where these effects start at $O\left(m_{e} \alpha^{6}\right)$ and therefore contribute in a much weaker manner to the theoretical prediction of the Lamb shift.

\subsubsection{QED corrections}

We will start with a brief review of the QED corrections that sum up to the first term in Eq. (8) and which are collected in Table 1, together with their power counting order and the potential term where they come from. For a detailed account of this computation see Ref. [11]. The first item in Table 1 is the largest contribution to this number. It comes from the electron vacuum polarization (VP) at $O\left(m_{r} \alpha^{3}\right)$, which is computed through the expectation value of the vacuum polarization potential $V_{V P}^{(0)}$. Note that in the analysis of hydrogen there is no such contribution.
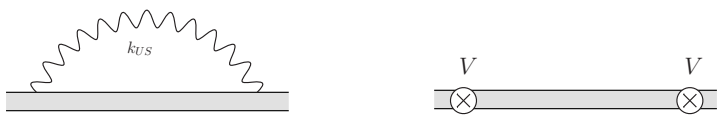

Figure 1. pNRQED diagrams depicting corrections to the bound state coming from the emission of an ultrasoft photon, in the first one and from two potential insertions in the second.

Items ii)-v) of Table 1 come from higher order contributions of the static potential, higher order corrections to the electron VP and also the light-by-light scattering contribution. We have collected them and computed them except for the last two items which can be found in Ref. [16, 17] and [18] respectively. Items vi) and viii) arise from relativistic corrections of the potential as well as the ultrasoft energy contribution pictured in the first diagram of Fig. 1. It is in these contributions that the dependence on the NRQED Wilson coefficients arises, therefore also giving place to the hadronic effects which are encoded within these coefficients. However, we split their contribution into pure QED and hadronic pieces. The relevant tree level diagrams with their explicit dependence on the Wilson coefficients are pictured in Fig. 2.

It is worth noting that in the computation of items iii), viii) and $\mathrm{x}$ ) in Table 1 one needs to use second order perturbation theory. This is pictured in the second diagram of Fig. 1. In order to compute this correction with two potential insertions we use the usual notation of quantum mechanics

$$
\delta E_{n l j}^{V \times V}=\left\langle\psi_{n l j}\left|V \frac{1}{\left(E_{n}-h\right)^{\prime}} V\right| \psi_{n l j}\right\rangle=\int \mathrm{d} \mathbf{r}_{2} \mathrm{~d} \mathbf{r}_{1} \psi_{n l j}^{*}\left(\mathbf{r}_{2}\right) V\left(\mathbf{r}_{2}\right) G_{n l}^{\prime}\left(\mathbf{r}_{1}, \mathbf{r}_{2}\right) V\left(\mathbf{r}_{1}\right) \psi_{n l j}\left(\mathbf{r}_{\mathbf{1}}\right)
$$


Table 1. Summary of the contributions to the muonic hydrogen $n=2$ Lamb shift coming from QED corrections.

\begin{tabular}{|c|c|c|c|}
\hline i) & $O\left(m_{r} \alpha^{3}\right)$ & $V_{\mathrm{vP}}^{(0)}$ & 205.00737 \\
\hline ii $)$ & $O\left(m_{r} \alpha^{4}\right)$ & $V_{\mathrm{VP}}^{(0)}$ & 1.50795 \\
\hline iii) & $O\left(m_{r} \alpha^{4}\right)$ & $V_{\mathrm{VP}}^{(0)}$ & 0.15090 \\
\hline iv) & $O\left(m_{r} \alpha^{5}\right)$ & $V_{\mathrm{VP}}^{(0)}$ & 0.00752 \\
\hline v) & $O\left(m_{r} \alpha^{5}\right)$ & $V_{\mathrm{LbL}}^{(0)}$ & $-0.00089(2)$ \\
\hline vi) & $O\left(m_{r} \alpha^{4} \times \frac{m_{\mu}^{2}}{m_{p}^{2}}\right)$ & $V^{(2,1)}+V^{(3,0)}$ & 0.05747 \\
\hline vii) & $O\left(m_{r} \alpha^{5}\right)$ & $V_{\mathrm{no}-\mathrm{VP}}^{(2,2)}+$ ultrasoft & -0.71896 \\
\hline viii $)$ & $O\left(m_{r} \alpha^{5}\right)$ & $V_{\mathrm{VP}}^{(2,2)}+V^{(2,1)} \times V_{\mathrm{VP}}^{(0,2)}$ & 0.01876 \\
\hline ix $)$ & $O\left(m_{r} \alpha^{6} \times \ln \left(\frac{m_{\mu}}{m_{e}}\right)\right)$ & $V^{(2,3)} ; c_{D}^{(\mu)}$ & -0.00127 \\
\hline x) & $O\left(m_{r} \alpha^{6} \times \ln \alpha\right)$ & $V_{\mathrm{VP}}^{(2,3)} ; c_{D}^{(\mu)}$ & -0.00454 \\
\hline
\end{tabular}
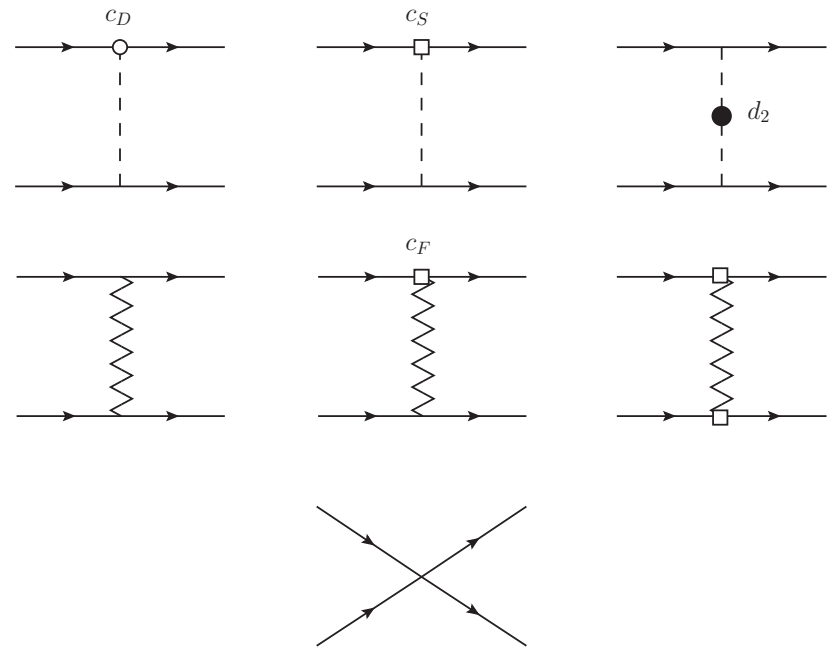

Figure 2. NRQED diagrams contributing to the relativistic corrections of the Lamb shift. Dashed lines and zigzag lines account for a longitudinal and a transversal photon respectively. The vertices proportional to the NRQED Wilson coefficients are explicitly named in the figure.

where the $G_{n l}^{\prime}$ is the reduced Green function (a suitable definition can be found in [11]).

Finally, the last two items in Table 1 are the large logarithmic contributions of the $O\left(m_{r} \alpha^{6}\right)$ correction. Their computation allows us to estimate the uncertainty of this computation by assuming it to be a $50 \%$ of their sum. 


\subsubsection{Hadronic effects}

The hadronic effects of the last two terms of Eq. (8) come from the NRQED Lagrangian

$$
\delta \mathcal{L}=\frac{d_{2}}{m_{p}^{2}} F_{\mu \nu} D^{2} F^{\mu \nu}-e \frac{c_{D}^{(p)}}{m_{p}^{2}} N_{p}^{\dagger} \nabla \cdot \mathbf{E} N_{p}+\frac{c_{3}}{m_{p}^{2}} N_{p}^{\dagger} N_{p} \mu^{\dagger} \mu
$$

from which the subsequent contribution to the $\delta^{(3)}(r)$-potential and the energy levels is

$$
\delta V^{\mathrm{had}}=\frac{D_{d}^{\mathrm{had}}}{m_{p}^{2}} \delta^{(3)}(\mathbf{r}), \quad \delta E_{n l}^{\mathrm{had}}=\frac{D_{d}^{\mathrm{had}}}{m_{p}^{2}} \frac{\left(m_{r} \alpha\right)^{3}}{\pi n^{3}} \delta_{l, 0},
$$

that depends on the following combination of the NRQED matching coefficients:

$$
D_{d}^{\mathrm{had}} \equiv-c_{3}^{\mathrm{had}}-16 \pi \alpha d_{2}^{\mathrm{had}}+\frac{\pi \alpha}{2} c_{D}^{\mathrm{had}}
$$

where $c_{3}^{\text {had }}$ accounts for two photon exchange contribution, $d_{2}^{\text {had }}$ for the hadronic vacuum polarization and, as noted in Eq. (9), $c_{D}^{\text {had }}$ encodes the definition of the proton radius.

All the hadronic contributions are collected in Table 2 together with their power counting order and the piece of the potential where they come from. The first two entries account for the contribution to the proton radius up to $O\left(m_{r} \alpha^{5}\right)$ and with the third one, which is the large logarithm of the $O\left(m_{r} \alpha^{6}\right)$, we can estimate the error (assuming, as for the QED part, a 50\%). Entry iv) is the hadronic VP, which can be extracted from experimental data via dispersion relations with a very small error. This was done by Jegerlehner [19], and this is the number we quote.

Table 2. Summary of the hadronic contributions to the Lamb shift in muonic hydrogen.

\begin{tabular}{|c|c|c|c|}
\hline i) & $O\left(m_{r} \alpha^{4} \times m_{r}^{2} r_{p}^{2}\right)$ & $V^{(2,1)} ; c_{D}^{(p)} ; r_{p}^{2}$ & $-5.19745 \frac{r_{p}^{2}}{\frac{\mathrm{fm}^{2}}{r_{p}^{2}}}$ \\
\hline ii) & $O\left(m_{r} \alpha^{5} \times m_{r}^{2} r_{p}^{2}\right)$ & $V_{\mathrm{VP}}^{(2,2)} ; c_{D}^{(p)} ; r_{p}^{2}$ & $-0.02815 \frac{r_{p}^{2}}{\mathrm{fm}^{2}}$ \\
\hline iii $)$ & $O\left(m_{r} \alpha^{6} \ln \alpha \times m_{r}^{2} r_{p}^{2}\right)$ & $V^{(2,3)} ; c_{D}^{(p)} ; r_{p}^{2}$ & $-0.00136 \frac{r_{p}^{2}}{\mathrm{fm}^{2}}$ \\
\hline vi $)$ & $O\left(m_{r} \alpha^{5} \times \frac{m_{r}^{2}}{m_{\rho}^{2}}\right)$ & $V_{\mathrm{VP}_{\text {had }}}^{(2)} ; d_{2}^{\text {had }}$ & $0.0111(2)$ \\
\hline v) & $O\left(m_{r} \alpha^{5} \times \frac{m_{r}^{2}}{m_{\rho}^{2}} \frac{m_{\mu}}{m_{\pi}}\right)$ & $V^{(2)} ; c_{3}^{\text {had }}$ & $0.0344(125)$ \\
\hline
\end{tabular}

Now let us focus on the two photon exchange contribution, which determines item v) of Table 2. Even if in our EFT setting it comes form a single Wilson coefficient of NRQED, it is customarily split into Born and polarizability terms. By introducing the contribution of the Delta particle, we can schematically write it in terms of its expansion parameters as

$$
c_{3}^{\mathrm{had}}=c_{3}^{\mathrm{Born}}+c_{3}^{\mathrm{pol}} \sim \alpha^{2} \frac{m_{\mu}}{m_{\pi}}\left(1+\# \frac{m_{\pi}}{\Delta}+\ldots\right)+O\left(\frac{\alpha^{2} m_{\mu}}{\Lambda_{\mathrm{Q} C D}}\right)
$$

In order to obtain this number we have computed the forward virtual Compton tensor in HBChPT up to $O\left(p^{3}\right)$. The diagrams that contribute to this computation are depicted in Fig. 3, both for the purely chiral contribution and for the contribution coming from the Delta resonance. 


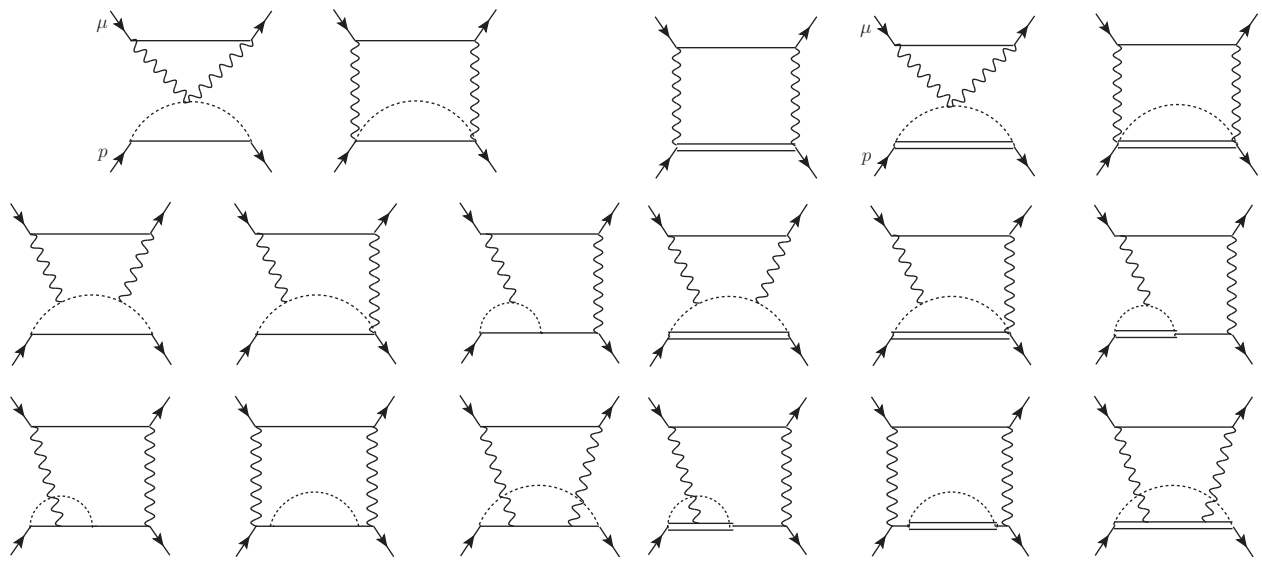

Figure 3. Diagrams contributing to the TPE in HBChPT. The eight leftmost diagrams are the pure chiral correction while the nine rightmost diagrams account for the contribution of the Delta particle. The dashed lines are pions, the wiggly lines are photons and the double lines depict the Delta excitation of the proton.

The forward virtual Compton tensor can be described in terms of four tensor functions as

$$
\begin{aligned}
T^{\mu \nu} & =\left(-g^{\mu \nu}+\frac{q^{\mu} q^{\nu}}{q^{2}}\right) S_{1}\left(\rho, q^{2}\right)+\frac{1}{m_{p}^{2}}\left(p^{\mu}-\frac{m_{p} \rho}{q^{2}} q^{\mu}\right)\left(p^{\nu}-\frac{m_{p} \rho}{q^{2}} q^{\nu}\right) S_{2}\left(\rho, q^{2}\right) \\
& -\frac{i}{m_{p}} \epsilon^{\mu \nu \rho \sigma} q_{\rho} s_{\sigma} A_{1}\left(\rho, q^{2}\right)-\frac{i}{m_{p}^{3}} \epsilon^{\mu \nu \rho \sigma} q_{\rho}\left(\left(m_{p} \rho\right) s_{\sigma}-(q \cdot s) p_{\sigma}\right) A_{2}\left(\rho, q^{2}\right)
\end{aligned}
$$

where the first line is the symmetric, spin-independent part that gives a contribution to the Lamb shift and the second line is the antisymmetric, spin-dependent piece, that contributes to the hyperfine splitting.

We find for the total TPE energy shift

$$
\delta E_{L}^{\mathrm{TPE}}=\frac{\left(m_{r} \alpha\right)^{3} c_{r}^{\mathrm{had}}}{8 \pi m_{p}^{2}}=\delta E_{L}^{\mathrm{Born}}+\delta E_{L}^{\mathrm{pol}}=28.59(\pi)+5.86(\pi \& \Delta)=34.4(12.5) \mu \mathrm{eV}
$$

In order to estimate the error of the quantities that arise from this computation we count $m_{\pi} \sim$ $\sqrt{\Lambda_{\mathrm{QCD}} /, m_{q}}$ and $\Delta \sim \frac{\Lambda_{\mathrm{QCD}}}{N_{c}}$, and so $\frac{m_{\pi}}{\Delta} \sim 1 / 2$.. Therefore, we associate a $50 \%$ uncertainty to the pure chiral computation. For all our computations we observe good convergence, the contribution due to the Delta being much smaller than the pure chiral result and well inside the $50 \%$ uncertainty. Leaving aside the Delta, the splitting with the next resonances suggest a mass gap of order $\Lambda_{\mathrm{QCD}} \sim$ 500-770 MeV depending on whether one considers the Roper resonance or the $\rho$. Therefore, we assign $\frac{m_{\pi}}{\Lambda_{\mathrm{QCD}}} \sim \frac{1}{3}$ and $\frac{\Delta}{\Lambda_{\mathrm{QCD}}} \sim \frac{1}{2}$, as the uncertainties of the pure chiral and the Delta related contributions respectively. We add these errors linearly for the final error. This gives the expected size of the uncomputed corrections but numerical factors may change the real size of the correction.

In order to compare to previous estimations of the TPE contribution we split our computation into Born and polarizability corrections as in the first equality of Eq. (13). 
The first term in Eq. (13) is generated by the spin-independent Born contribution to $T^{\mu v}$ in Eq. (14). At leading order in the NR expansion it reads

$$
c_{3, \text { Born }}=4(4 \pi \alpha)^{2} m_{p}^{2} m_{\mu} \int \frac{d^{D-1} q}{(2 \pi)^{D-1}} \frac{1}{\mathbf{q}^{6}} G_{E}^{(0)} G_{E}^{(2)}\left(-\mathbf{q}^{2}\right)=\frac{\pi}{3} \alpha^{2} m_{p} m_{\pi}\left\langle r^{3}\right\rangle_{(2)},
$$

where the HBChPT electromagnetic form factors had already been computed in $[3,20,21]$. In the last equality it can be seen that this contribution can be related to (one of) the Zemach moments, so we profit our computation to study the EFT prediction for the different Zemach momenta, which we summarize in Table 3.

Table 3. Values of hr $\mathrm{n}$ i in fermi units. The first two rows give the prediction from the effective theory: the first with only pions and the second with pions and Deltas. The third row corresponds to the standard dipole fit with

$\left\langle r^{2}\right\rangle=0.6581 \mathrm{fm}^{3}$. The fourth and fifth rows correspond to different parameterizations of experimental data

\begin{tabular}{|l||c|c|c|c|c|c|}
\hline$(\mathrm{fm})$ & $\left\langle r^{3}\right\rangle$ & $\left\langle r^{4}\right\rangle$ & $\left\langle r^{5}\right\rangle$ & $\left\langle r^{6}\right\rangle$ & $\left\langle r^{7}\right\rangle$ & $\left\langle r^{3}\right\rangle_{(2)}$ \\
\hline \hline$\pi$ & 0.4980 & 0.6877 & 1.619 & 5.203 & 20.92 & 0.9960 \\
$\pi \& \Delta$ & 0.4071 & 0.6228 & 1.522 & 4.978 & 20.22 & 0.8142 \\
\hline Dipole [22] & 0.7706 & 1.083 & 1.775 & 3.325 & 7.006 & 2.023 \\
Kelly [23] & 0.9838 & 1.621 & 3.209 & 7.440 & 19.69 & 2.526 \\
Distler et al[24] & $1.16(4)$ & $2.59(19)(04)$ & $8.0(1.2)(1.0)$ & $29.8(7.6)(12.6)$ & - & $2.85(8)$ \\
\hline
\end{tabular}

The chiral prediction is expected to give the dominant contribution of $\left\langle r^{n}\right\rangle$ for $n \geq 3$. For $n=2$ it could also give the leading chiral logarithm. For smaller $n$ the chiral corrections are subleading. Nevertheless, we observe large differences, bigger than the errors, not only in the EFT prediction but also in the predictions from different fits. In this respect, we believe that the chiral result may help to shape the appropriate fit function and, thus, to discriminate between different options, as well as to assess uncertainties.

Finally, for the Born energy shift we compare our result to other determinations from dispersion relations in Table 4. Note that the correction associated to the Delta is much smaller and so the EFT result shows a good convergence. On the other hand our result strongly differs with respect to standard values obtained from dispersion relations.

Table 4. Predictions for the Born contribution to the $n=2 \mathrm{Lamb}$ shift. The first two entries correspond to dispersion relations. The last two entries are the predictions of HBET, for only pions and including the Delta.

\begin{tabular}{|c|ccc|ccc|}
\hline$(\mu \mathrm{eV})$ & DR: & Pachucki [25] & Carlson [26] & HBET & $(\pi)$ & $(\pi \& \Delta)$ \\
\hline$\delta E_{L}^{\text {Born }}$ & & $23.2(1.0)$ & $24.7(1.6)$ & & $10.1(5.1)$ & $8.3(4.3)$ \\
\hline
\end{tabular}

We now look at the contribution from the second term in Eq. (13), which can be obtained from the polarizability piece of the symmetric spin-independent piece of $T^{\mu v}$ in Eq. (14) in the following way

$$
\begin{aligned}
c_{3}^{\mathrm{pol}}= & -e^{4} m_{p} m_{l_{i}} \int \frac{d^{4} k_{E}}{(2 \pi)^{4}} \frac{1}{k_{E}^{4}} \frac{1}{k_{E}^{4}+4 m_{\mu}^{2} k_{0, E}^{2}} \\
& \times\left\{\left(3 k_{0, E}^{2}+\mathbf{k}^{2}\right) S_{1}^{\mathrm{pol}}\left(i k_{0, E},-k_{E}^{2}\right)-\mathbf{k}^{2} S_{2}^{\mathrm{pol}}\left(i k_{0, E},-k_{E}^{2}\right)\right\}+O\left(\alpha^{3}\right) .
\end{aligned}
$$

In Table 5 we compare our determination with previous results. Most of them are obtained by a combination of dispersion relations plus some modeling of the subtraction term that we discuss 
below. The analysis of Ref.[27] has a different status. In this reference the polarizability correction was computed using $\mathrm{B} \chi \mathrm{PT}$ with only pions. Such computation treats the baryon relativistically. The result incorporates some subleading effects, which are sometimes used to give an estimate of higher order effects in HBChPT. Nevertheless, the computation also assumes that a theory with only baryons and pions is appropriate at the proton mass scale. This should be taken with due caution. Still, it would be desirable to have a deeper theoretical understanding of this difference, which may signal that relativistic corrections are important for the polarizability correction. In any case, the $\mathrm{B} \chi \mathrm{PT}$ computation differs from our chiral result by around $50 \%$, which we consider reasonable.

Table 5. Predictions for the polarizability contribution to the $n=2$ Lamb shift. The first four entries use dispersion relations for the inelastic term and different modeling functions for the subtraction term. The 5th entry is obtained using $\mathrm{B} \chi \mathrm{PT}$. The last two are our HBET predictions for only pions including the Deltas

\begin{tabular}{|l|cccc|c|cc|}
\hline & DR+Model & & & & $\mathrm{B} \chi \mathrm{PT}(\pi)$ & $\mathrm{HBET}(\pi)$ & $(\pi \& \Delta)$ \\
& $\begin{array}{c}\text { Pachucki } \\
{[28]}\end{array}$ & $\begin{array}{c}\text { Martynenko } \\
{[29]}\end{array}$ & $\begin{array}{c}\text { Carlson et al } \\
{[26]}\end{array}$ & $\begin{array}{c}\text { Gorchtein et al } \\
\text { Alarcon et al } \\
\text { Nevado et al }\end{array}$ & \\
& {$[27]$} & {$[31]$} & \\
\hline$\delta E_{L}^{\mathrm{pol}}(\mu \mathrm{eV})$ & $12(2)$ & 11.5 & $7.4(2.4)$ & $15.3(5.6)$ & $8.2\left(_{-2.5}^{+1.2}\right)$ & $18.5(9.3)$ & $26.2(10.0)$ \\
\hline
\end{tabular}

We would like to emphasize at this point that this result is a pure prediction of the EFT. It is also the most precise expression that can be obtained in a model independent way, since $O\left(m_{r} \alpha^{5} \frac{m_{\mu}^{3}}{\Lambda_{\mathrm{OCD}}^{3}}\right)$ effects are not controlled by the chiral theory and would require new counterterms. Note also that the TPE contribution agrees better with DR results than Born and polarizability do separately.

\subsection{The hyperfine splitting}

From the results for the forward virtual Compton tensor [32], we can compute the EFT contribution of the TPE to the hyperfine (HF) splitting in muonic hydrogen allowing for comparison with both previous estimates and experimental data. A detailed account of this analysis can be found in [12].

The TPE in HF splitting is related to the NRQED lagrangian

$$
\delta \mathcal{L}=-\frac{c_{4}^{p l_{i}}}{m_{p}^{2}} N_{p}^{\dagger} \sigma N_{p} l^{\dagger} \sigma l
$$

where

$$
c_{4}^{p l_{i}} \equiv \alpha^{2} c_{4, \mathrm{TPE}}^{p l_{i}}+\alpha^{3} \delta c_{4}^{p l_{i}} .
$$

From the Lagrangian in Eq. (18) we obtain the subsequent contribution to the $\delta^{(3)}(r)$-potential and the energy levels read

$$
\delta V=\frac{2 c_{4}^{p l_{i}}}{m_{p}^{2}}\left(\mathbf{S}_{\mathbf{1}}+\mathbf{S}_{\mathbf{2}}\right)^{2} \delta^{(3)}(\mathbf{r}), \quad \Delta E_{H F}=\frac{4\left(\alpha m_{r}\right)^{3} c_{4}^{p l_{i}}}{n^{3} \pi m_{p}^{2}} .
$$

We can obtain the TPE Wilson coefficient from the antisymmetric spin-dependent piece of the $T^{\mu \nu}$ tensor in Eq. (14), such that

$$
c_{4, \mathrm{TPE}}^{p l_{i}}=\frac{1}{3 \pi^{2}} \int d^{4} k \frac{1}{k_{E}^{2}} \frac{1}{k_{E}^{4}+4 m_{l_{i}}^{2} k_{0, E}^{2}}\left\{A_{1}\left(i k_{0, E},-k_{E}^{2}\right)\left(k_{0, E}^{2}+2 k_{E}^{2}\right)+i 3 k_{E}^{2} \frac{k_{0, E}}{m_{p}} A_{2}\left(i k_{0, E},-k_{E}^{2}\right)\right\} .
$$




\subsubsection{The HF splitting in hydrogen}

The hyperfine splitting of the ground state of hydrogen is one of the most accurate measurements made by mankind $[33,34]$. Upon comparison of this result to the QED contributions which are (partially) known up to $O\left(m_{e} \alpha^{8}\right)$ and the inclusion of the leading recoil corrections, we can obtain a prediction of the TPE hyperfine energy shift

$$
E_{\text {hyd, } \mathrm{HF}}^{\text {exp }}(1 S)-E_{\text {hyd, } \mathrm{HF}}^{\mathrm{QED}}(1 S)-E_{\text {hyd, HF }}^{\mathrm{QED}, \text { recoil }}(1 S)=-46.94 \mathrm{kHz},
$$

from which we extract for the TPE piece of the Wilson coefficient

$$
c_{4, \mathrm{TPE}}^{p e}=-48.7(5),
$$

where the error is the estimate of the corrections associated to the order $\alpha$, which is taken to be $1 \%$.

\subsubsection{The HF splitting in muonic hydrogen}

In the case of muonic hydrogen, there are two ways in which we can obtain a prediction for the TPE correction to the HF splitting. We can do so by comparison to experimental data or by using the result for hydrogen plus the computation of the HBChPT forward virtual Compton tensor.

The experimental measurement the (2S) HF splitting has already been obtained [7], and an experimental measurement for the (1S) HF splitting is foreseen for the next year. Analogously to what we have presented for the Lamb shift, in Tables I and II of Ref. [12], the QED contributions to the (1S) and $(2 \mathrm{~S})$ hyperfine splitting are presented. These corrections comprise the effect of electron VP, second order perturbation theory and the contribution coming from relativistic corrections. They include a partial set of the $O\left(m_{r} \alpha^{6}\right)$ contribution.

Upon comparison of the experimental measurement of the (2S) HF splitting to our theoretical prediction we obtain (preliminary):

$$
c_{4, \mathrm{TPE}}^{p \mu}=-\alpha^{2} 45.5(0.1)_{\mathrm{t} h}(1.6)_{\exp } \quad E_{\mu p, H F}^{\mathrm{TPE}}=0.1456(51)_{\exp }(2)_{\mathrm{th}} .
$$

The errors are dominated by experiment, on the other hand, the foreseen determination of the hyperfine of the ground state would eliminate most of this error.

We can also obtain the TPE Wilson coefficient by taking the difference of the muonic hydrogen and electronic hydrogen coefficients such that

$$
c_{4, \mathrm{TPE}}^{p \mu}=c_{4, \mathrm{TPE}}^{p e}+\left[c_{4, \mathrm{TPE}}^{p \mu}-c_{4, \mathrm{TPE}}^{p e}\right]+O(\alpha) .
$$

Each coefficient can be split into terms coming from different pieces of $T^{\mu v}$ in Eq. (14):

$$
c_{4, \mathrm{TPE}}^{p l}=c_{4, \mathrm{R}}^{p l}+c_{4, \mathrm{point}-\mathrm{like}}^{p l}+c_{4, \mathrm{Born}}^{p l}+c_{4, \mathrm{pol}}^{p l} .
$$

Since $c_{4, \mathrm{R}}^{p l_{i}} \simeq c_{4, \mathrm{R}}^{p}$ up to terms of $O\left(\alpha^{2} m_{l_{i}} / \Lambda_{\mathrm{QCD}}\right)$, we can obtain the following relation

$$
c_{4, \mathrm{TPE}}^{p \mu}-c_{4, \mathrm{TPE}}^{p e}=\left[c_{4, \mathrm{Born}}^{p \mu}-c_{4, \mathrm{Born}}^{p e}\right]+\left[c_{4, \text { point-like }}^{p \mu}-c_{4, \mathrm{point}-\mathrm{like}}^{p e}\right]+\left[c_{4, \mathrm{pol}}^{p \mu}-c_{4, \mathrm{pol}}^{p e}\right] .
$$

By considering differences in Eq. (27) the ultraviolet behavior gets regulated and the logarithmic divergences vanish. This makes these contributions to be very small an negligible compared with the uncertainties. For the point-like contribution we obtain

$$
c_{4, \text { point-like }}^{p \mu}-c_{4, \text { point-like }}^{p e}=\left(1-\frac{\kappa_{p}^{2}}{4}\right) \ln \frac{m_{\mu}^{2}}{m_{e}^{2}}+\frac{m_{\mu}^{2}}{m_{p}^{2}}\left(1+\frac{\kappa_{p}}{2}\left(1-\frac{\kappa_{p}}{6}\right)\right) \ln \left(\frac{m_{\mu}^{2}}{v_{\text {chiral }}^{2}}\right) \simeq 2.09-0.09=2.00(9),
$$


where we use the $\frac{m_{\mu}}{m_{p}^{2}}$ logarithmic term for the estimate of subleading terms setting the factorization scale to the proton mass.

For the polarizability we obtain (note that this term vanishes in the large $N_{c}$ limit, except for the tree-level-like contribution)

$$
c_{4, \mathrm{pol}}^{p \mu}-c_{4, \mathrm{pol}}^{p e}= \begin{cases}0.17(9) & (\pi), \\ 0.25(10) & (\pi \& \Delta),\end{cases}
$$

For the Born term we get zero at leading order in the $m_{l}$ expansion. The first nontrivial term is quadratic in the lepton mass. We obtain

$$
\begin{aligned}
& c_{4, \text { Born }}^{p \mu}-c_{4, \text { Born }}^{p e}=-\int_{0}^{\infty} d p \frac{1}{3 p} G_{M}^{(1)}\left(-p^{2}\right) \\
& \times\left[\left(\frac{p^{2} \kappa_{p}}{m_{\mu}^{2}}+\frac{32 m_{\mu}^{4}-8 m_{\mu}^{2} p^{2}\left(\kappa_{p}+2\right)-2 p^{4} \kappa_{p}}{m_{\mu}^{2} p\left(\sqrt{4 m_{\mu}^{2}+p^{2}}+p\right)}+3 \kappa_{p}+8\right)-\left(m_{\mu} \rightarrow m_{e}\right)\right]
\end{aligned}
$$

This integral is finite. The high energy behavior is cut by the cancellation between the integrands of the muon and electron in such a way that the ultraviolet behavior of the integrand scales as $1 / p^{2}$ (while $G_{M}^{(1)}\left(-p^{2}\right) \sim p$ for large $p$ ). The integrand in the limit $p \rightarrow 0$ is also finite since $G_{M}^{(1)}\left(-p^{2}\right) \sim p^{2}$ for small $p$. After the numerical evaluation we find

$$
c_{4, \mathrm{Born}}^{p \mu}-c_{4, \mathrm{Born}}^{p e}= \begin{cases}0+1.11(55) & (\pi), \\ 0+1.42(53) & (\pi \& \Delta),\end{cases}
$$

Both for the polarizability and Bron differences in Eqs. (29) and (32) we use the same error analysis explained in Section 2.1.2 for the Lamb shift.

Overall we obtain the difference

$$
c_{4, \mathrm{TPE}}^{p \mu}-c_{4, \mathrm{TPE}}^{p e}=3.67(72),
$$

leading to a prediction for the Wilson coefficient and the TPE energy shift

$$
c_{4, T P E}^{p \mu}=-45.02(1.3) \quad \delta E_{\mathrm{TPE}}^{p \mu}=-\frac{1}{n^{3}} 1.15(3) \mathrm{meV} .
$$

This result is in good agreement with the one obtained by comparison to experiment in Eq. (24).

\section{Conclusions}

We develop the $\mathrm{N}^{3} \mathrm{LO}$ NRQED potential for different masses. We apply this computation to muonic hydrogen, and in particular to its Lamb shift and hyperfine splitting, for which we obtain a theoretical prediction in a model independent way in an EFT framework. The main source of uncertainty in both predictions is the TPE, which we predict by computing the forward virtual Compton tensor in HBET up to $O\left(p^{3}\right)$.

From our prediction of the TPE contribution to the Lamb shift we observe that the EFT Born and polarizability contributions are separately different from the results obtained by means of dispersion relations and modeling. Nevertheless, out total prediction for the TPE energy shift is in good agreement with these computations. This is an outcome of the EFT analysis that should be further 
understood. Upon comparison of our theoretical prediction of the Lamb shift to experiment, we can extract a value for the proton charge radius of $r_{p}=0.8775(51) \mathrm{fm}$ which is $6.8 \sigma$ away from the combined CODATA value. Our prediction gives model independent significance to this discrepancy.

We use the same computation of the forward virtual Compton tensor to obtain a prediction of the TPE contribution HF splitting in muonic hydrogen, by calculating its difference to the same splitting in electronic hydrogen. Our result is in good agreement with the one extracted from the experimental data for the (2S) splitting. Future experimental data of the (1S) HF splitting will decrease the experimental error of the latter, allowing for a more demanding comparison with out EFT result.

To conclude, let us note physics of muonic atoms is one of the most interesting topics nowadays, with experiments such as PSI, MAMI, JLAB, MUSE, etc. prepared to or in the process of performing measurements of different states, both to disentangle the proton radius puzzle and to obtain new data of muonic systems, where hadronic physics plays a major role.

\section{References}

[1] S. Weinberg, Phys. Rev. 166, 1568 (1968)

[2] E.E. Jenkins, A.V. Manohar, Phys. Lett. B255, 558 (1991)

[3] V. Bernard, N. Kaiser, J. Kambor, U.G. Meissner, Nucl. Phys. B388, 315 (1992)

[4] W.E. Caswell, G.P. Lepage, Phys. Lett. B167, 437 (1986)

[5] A. Pineda, J. Soto, Nucl. Phys. Proc. Suppl. 64, 428 (1998), hep-ph/9707481

[6] R. Pohl et al., Nature 466, 213 (2010)

[7] A. Antognini, F. Kottmann, et al., Annals Phys. 331, 127 (2013), 1208. 2637

[8] R. Pohl et al. (2016), 1609.03440

[9] C. Peset, A. Pineda, Eur. Phys. J. A51, 32 (2015), 1403. 3408

[10] C. Peset, A. Pineda, Nucl. Phys. B887, 69 (2014), 1406.4524

[11] C. Peset, A. Pineda, Eur. Phys. J. A51, 156 (2015), 1508.01948

[12] C. Peset, A. Pineda (2016), 1612.xxxxx

[13] A. Pineda, Phys. Rev. C71, 065205 (2005), hep-ph/0412142

[14] A. Antognini et al., Science 339, 417 (2013)

[15] P.J. Mohr, B.N. Taylor, D.B. Newell, Rev. Mod. Phys. 84, 1527 (2012), 1203.5425

[16] T. Kinoshita, M. Nio, Phys. Rev. Lett. 82, 3240 (1999), [Erratum: Phys. Rev. Lett.103,079901(2009)], hep-ph/9812442

[17] V.G. Ivanov, E.Yu. Korzinin, S.G. Karshenboim (2009), 0905.4471

[18] S.G. Karshenboim, E.Yu. Korzinin, et al., JETP Lett. 92, 8 (2010), 1005 . 4880

[19] F. Jegerlehner, Nucl. Phys. Proc. Suppl. 51C, 131 (1996), hep-ph/9606484

[20] J. Gasser, M.E. Sainio, A. Svarc, Nucl. Phys. B307, 779 (1988)

[21] V. Bernard, H.W. Fearing, T.R. Hemmert, U.G. Meissner, Nucl. Phys. A635, 121 (1998), [Erratum: Nucl. Phys.A642,563(1998)], hep-ph/9801297

[22] T. Janssens, R. Hofstadter, E.B. Hughes, M.R. Yearian, Phys. Rev. 142, 922 (1966)

[23] J.J. Kelly, Phys. Rev. C70, 068202 (2004)

[24] M.O. Distler, J.C. Bernauer, T. Walcher, Phys. Lett. B696, 343 (2011), 1011. 1861

[25] K. Pachucki, Phys. Rev. A60, 3593 (1999)

[26] C.E. Carlson, M. Vanderhaeghen, Phys. Rev. A84, 020102 (2011), 1101.5965

[27] J.M. Alarcon, V. Lensky, V. Pascalutsa, Eur. Phys. J. C74, 2852 (2014), 1312 . 1219

[28] K. Pachucki, Phys. Rev. A53, 2092 (1996) 
[29] A.P. Martynenko, Phys. Atom. Nucl. 69, 1309 (2006), hep-ph/0509236

[30] M. Gorchtein, et al., Phys. Rev. A87, 052501 (2013), 1302.2807

[31] D. Nevado, A. Pineda, Phys. Rev. C77, 035202 (2008), 0712 . 1294

[32] X.D. Ji, J. Osborne, J. Phys. G27, 127 (2001), hep-ph/9905410

[33] H. Hellwig, R.F.C. Vessot, M.W. Levine, et al., IEEE Trans. IM-19, 200 (1970)

[34] L. Essen, R.W. Donaldson, M.W. Bangham, et al., Nature 229, 110 (1971) 